\title{
Community norms of the Muscle Dysmorphic Disorder Inventory (MDDI) among gender minority populations
}

\author{
Jason M. Nagata ${ }^{1 *}$ (D), Emilio J. Compte ${ }^{2,3}$, F. Hunter McGuire ${ }^{4}$, Jason M. Lavender ${ }^{5,6}$, Tiffany A. Brown ${ }^{7,8}$, \\ Stuart B. Murray ${ }^{9}$, Annesa Flentje ${ }^{10,11,12}$, Matthew R. Capriotti ${ }^{13,12}$, Micah E. Lubensky ${ }^{10,12}$, \\ Juno Obedin-Maliver ${ }^{12,14,15}$ and Mitchell R. Lunn ${ }^{12,15,16}$
}

\begin{abstract}
Purpose: Representing the pathological extreme pursuit of muscularity, muscle dysmorphia (MD) is characterized by a pervasive belief or fear around insufficient muscularity and an elevated drive for muscularity. Despite evidence of heightened body image-related concerns among gender minority populations, little is known about the degree of MD symptoms among gender minorities, particularly based on Muscle Dysmorphic Disorder Inventory (MDDI) scores. The objective of this study was to assess community norms of the MDDI in gender-expansive people, transgender men, and transgender women.

Method: Data from participants in The PRIDE Study, an existing study of health outcomes in sexual and gender minority people, were examined. We calculated means, standard deviations, and percentiles for the MDDI total and subscale scores among gender-expansive people (i.e., those who identify outside of the binary system of man or woman; $n=1023)$, transgender men $(n=326)$, and transgender women $(n=177)$. The Kruskal-Wallis test was used to assess group differences and post hoc Dunn's tests were used to examine pairwise differences.

Results: Transgender men reported the highest mean MDDI total score $(30.5 \pm 7.5)$, followed by gender-expansive people (27.2 \pm 6.7$)$, then transgender women $(24.6 \pm 5.7)$. The differences in total MDDI score were driven largely by the Drive for Size subscale and, to a lesser extent, the Functional Impairment subscale. There were no significant differences in the Appearance Intolerance subscale among the three groups.

Conclusions: Transgender men reported higher Drive for Size, Functional Impairment, and Total MDDI scores compared to gender-expansive people and transgender women. These norms provide insights into the experience of MD symptoms among gender minorities and can aid researchers and clinicians in the interpretation of MDDI scores among gender minority populations.
\end{abstract}

\footnotetext{
* Correspondence: jasonmnagata@gmail.com

'Department of Pediatrics, University of California, 550 16th Street, 4th Floor, Box 0110, San Francisco, CA 94158, USA

Full list of author information is available at the end of the article
}

(c) The Author(s). 2021 Open Access This article is licensed under a Creative Commons Attribution 4.0 International License, which permits use, sharing, adaptation, distribution and reproduction in any medium or format, as long as you give appropriate credit to the original author(s) and the source, provide a link to the Creative Commons licence, and indicate if changes were made. The images or other third party material in this article are included in the article's Creative Commons licence, unless indicated otherwise in a credit line to the material. If material is not included in the article's Creative Commons licence and your intended use is not permitted by statutory regulation or exceeds the permitted use, you will need to obtain permission directly from the copyright holder. To view a copy of this licence, visit http://creativecommons.org/licenses/by/4.0/. The Creative Commons Public Domain Dedication waiver (http://creativecommons.org/publicdomain/zero/1.0/) applies to the data made available in this article, unless otherwise stated in a credit line to the data. 


\section{Plain English summary}

Transgender people have a gender identity or expression that differs from what is typically associated with the sex assigned to them at birth. Gender-expansive refers to gender identities that do not fit within the binary (woman/ man) gender system. We asked gender-expansive people, transgender men, and transgender women in The PRIDE Study to fill out a widely used survey about muscle dysmorphia, a condition in which a person worries they are not muscular enough. The results showed that transgender men had the highest muscle dysmorphia symptoms, followed by gender-expansive people, then transgender women. These findings can help clinicians and researchers in interpreting a survey assessing muscle dysmorphia in gender-expansive people, transgender men, and transgender women. Characterizing muscle dysmorphia symptoms in these populations is an important step to improve mental wellness among transgender and gender-expansive people.

Keywords: Muscle dysmorphia, Muscle dysmorphic disorder, Body image, Body dissatisfaction, Body dysmorphia, Transgender, Gender non-conforming, Genderqueer, Gender minority, LGBTQ

\section{Introduction}

Muscle dysmorphia (MD), a specifier of Body Dysmorphic Disorder (BDD) in the Fifth Edition of the Diagnostic and Statistical Manual of Mental Disorders (DSM-5), is characterized by an excessive preoccupation with muscularity and the belief that one's body or body parts are insufficiently muscular [1]. MD involves a host of social and functional impairments arising from timeconsuming behavioral aspects of MD (e.g., excessive exercise, mirror checking, disordered eating behaviors) and shame about perceived physical appearance flaws [2-4]. In addition, individuals with MD symptoms often have comorbid psychiatric diagnoses and symptoms, including mood and anxiety disorders, substance use disorders, suicidal behaviors, and disordered eating behaviors $[2,3$, 5]. These impairments highlight the need to investigate populations that may be at higher risk for the development of MD symptoms, including gender minorities.

Most prior MD research has been conducted using samples of cisgender men (i.e., individuals who identify as a man and were assigned male at birth) $[3,6,7]$. As a result, there is limited empirical knowledge about experiences of $\mathrm{MD}$ among people with other genders and gender identities, including transgender and genderexpansive (TGE) populations. Transgender people have a gender identity or expression that differs from those traditionally associated with the sex assigned to them at birth. (See Table 1 for additional clarification on assigned sex at birth and gender identity among transgender men and transgender women, respectively.) Gender-expansive refers to a spectrum of gender identities which exist outside the gender binary (i.e., man, woman), including, but not limited to, individuals with no particular or multiple gender identities and those whose gender identity shifts over time or in different contexts. The current study reports on transgender men, transgender women, and gender-expansive individuals. For the purposes of this research, transgender men and transgender women indicated having a gender that aligned with the gender binary, while gender-expansive individuals indicated having a gender or genders that exist outside of the gender binary.

Stemming from individual, interpersonal, and structural stigma, the higher prevalence of adverse health outcomes among TGE populations relative to cisgender populations is well-documented [8]. In particular, the gender minority stress framework posits that TGE people may be more likely to experience poor psychological health due to a variety of social factors, including internalized transphobia (i.e., discomfort with one's TGE identity arising from an internalization of societal gender norms that privilege cisgender gender identities and

Table 1 Explanation of Classification of Participants

\begin{tabular}{lll}
\hline Population & Gender identity & Sex assigned at birth \\
\hline Cisgender man & man (exclusively) & male \\
Cisgender woman & woman (exclusively) & female \\
Transgender man & man, transgender man, or transmasculine (write-in) & female \\
Transgender woman & woman, transgender woman, or transfeminine (write-in) & male \\
Gender-expansive person & Included: genderqueer, multiple gender identities, another gender identity, non-binary, nonconforming, genderfluid, inter- \\
& sex, two-spirit, agender, bigender. This category included anyone not classified as cisgender man, cisgender woman, trans- & \\
\hline
\end{tabular}

ancludes any combination of man, transgender man, and/or transmasculine, but not other gender identities

${ }^{b}$ Includes any combination of woman, transgender woman, and/or transfeminine, but not other gender identities 
expressions), gender-related discrimination, and gender identity non-affirmation $[9,10]$.

Due to diverse social experiences and communityspecific body image norms, gender minority communities are not monolithic, and some groups may be at differential risk for the development of MD symptoms. Emerging research conducted in Italy indicates that transgender men report an equally high drive for muscularity as cisgender men, with nonbinary individuals, transgender women, and cisgender women reporting lower muscularity concerns [11]. Similar to cisgender women, transgender women may experience lower muscularity concerns due to feminine body norms placing greater emphasis on thinness [12]. Moreover, genderexpansive individuals may be disproportionately exposed to certain social stressors and psychological comorbidities that, in turn, may place them at higher risk for body image concerns. For instance, relative to cisgender and binary transgender men and women, gender-expansive individuals report higher lifetime experiences of psychological distress and self-harm behaviors, as well as being targeted for interpersonal violence and discrimination such as sexual assault and harassment [13]. Taken together, these diverse factors highlight the importance of the nature and severity of MD symptoms across different gender minority groups.

Though other MD symptom measures exist (e.g., the Muscle Dysmorphia Inventory [14], the Muscle Appearance Satisfaction Scale [15]), the Muscle Dysmorphic Disorder Inventory (MDDI) [16] was chosen for the current study, as it is one of the most widely used questionnaires assessing MD symptoms [7]. The MDDI has been psychometrically evaluated in samples of mostly cisgender men from diverse geographic locations and in multiple languages [17-23], and has received psychometric support specifically in samples of cisgender gay men and lesbian women [24]. One recent study used the MDDI to examine MD symptoms among TGE participants from Italy [11]; however, this investigation was limited by a small sample of TGE individuals and did not generate norms for the measure. No prior research has examined the nature and severity of MD symptoms and reported MDDI norms among gender-expansive individuals, transgender men, and transgender women from the community in the United States. Understanding MD symptoms in these populations is critical to the development of gender-inclusive clinical and public health interventions for preventing or mitigating muscularity-oriented body image concerns and related behavioral symptoms (e.g., excessive exercise, disordered eating). Therefore, the purpose of the current study is to describe community norms of the MDDI in three gender minority populations from the United States and to compare MDDI scores across these populations. Consistent with findings from prior research on TGE individuals [14], it was expected that MD symptoms would be highest among transgender men.

\section{Methods}

The current study is based on data from a subsample of participants in The Population Research in Identity and Disparities for Equality (PRIDE) Study. The PRIDE Study is a national online longitudinal cohort study that examines the health and wellbeing of sexual and gender minority adults in the United States. From April 2018 to August 2018, 4285 participants from The PRIDE Study completed a single cross-sectional 'Eating and Body Image' survey via any web-enabled device. More information about The PRIDE Study's research design and recruitment procedures can be found elsewhere $[25,26]$.

\section{Inclusion Criteria \& Study Population}

Inclusion criteria for the current study were completing the 'Eating and Body Image' survey (at least $50 \%$ of the MDDI, age, and body mass index [BMI] questions); living in the United States or its territories (e.g., Puerto Rico); being aged 18 years or older; being able to read and respond to an English-language survey; and being classified as a gender-expansive person, transgender man, or transgender woman.

Of the 4282 participants from The PRIDE Study, 1653 (38.6\%) identified as either a gender-expansive person, a transgender man, or a transgender woman. Of these 1653 participants, 127 (7.7\%) were excluded because they completed less than half of the relevant survey items (MDDI) and/or critical characteristics (age, BMI). Among gender subgroups, this resulted in 97 (8.7\%) gender-expansive individuals, 26 (7.4\%) transgender men, and four (2.2\%) transgender women being excluded. The final sample included 1526 participants who were classified as gender-expansive people $(N=1023$; $67.0 \%)$, transgender men $(N=326 ; 21.4 \%)$, or transgender women $(N=177 ; 11.6 \%)$.

\section{Participant Recruitment \& Informed Consent}

Participant recruitment occurred through several methods: community engagement through PRIDEnet (a national network of organizations and individuals to engage sexual and gender minority communities), online advertising through blog posts and newsletters, distribution of The PRIDE Study-branded promotional items, conference and event-based outreach, advertisements on social media, and word-of-mouth. Data were collected on a cloud-based, secure, digital platform [26]. Participants could access the survey platform from any smartphone, tablet, or computer. Compensation was not provided for completing the survey. This study was approved by the Stanford University and University of California, San Francisco Institutional Review Boards, as 
well as The PRIDE Study's Research Advisory Committee and Participant Advisory Committee. All participants provided written informed consent to participate.

\section{Measures}

\section{Sociodemographic questionnaire}

Participants self-reported sociodemographic information, including race, ethnicity, age, country of birth, and education level. We calculated BMI based on self-reported weight and height [weight $(\mathrm{kg}) /$ height $(\mathrm{m})^{2}$ ].

\section{Gender Identity \& sex Assigned at birth}

Participants were able to indicate their current gender identity (check all that apply) with options of "Man," "Genderqueer," "Transgender man," "Transgender woman," "Woman," or "Another gender identity" (with the option to specify). Participant's sex assigned at birth was assessed with the question "What sex were you assigned at birth on your original birth certificate?" with options of "Female" or "Male." Table 1 describes the classification rules that were applied to form the final samples. Participants in the current study were classified as either: (1) transgender man (man/transgender man/ transmasculine [write-in] gender identity and female sex assigned at birth); (2) transgender woman (woman/ transgender woman/transfeminine [write-in] gender identity and male sex assigned at birth); or (3) genderexpansive people including genderqueer, multiple gender identities, another gender identity, non-binary, nonconforming, genderfluid, intersex, two-spirit, agender, and bigender (anyone not classified as a cisgender man, cisgender woman, transgender man, or transgender woman).

\section{Muscle dysmorphic disorder inventory (MDDI)}

The MDDI is a 13-item measure designed to assess symptoms of muscle dysmorphia with individual items rated on a Likert-type scale from 1 (never) to 5 (always) [16]. The measure provides a total score and three subscale scores: Drive for Size (DFS; 5 items, range 5-25), Appearance Intolerance (AI; 4 items, range 4-20), and Functional Impairment (FI; 4 items, range 4-20). Items are summed to generate the total score and subscale scores, with higher values reflecting a greater severity of MD symptoms. The MDDI has demonstrated evidence of reliability and validity in samples of college-aged men [16] and sexual minority men and women [24, 27]. For participants in this study, item five ("I think my chest is too small") was modified to specify "chest (muscle)", so as to not confuse "chest" with breast size [24]. The MDDI total and subscale scores demonstrated acceptable to good internal consistency for gender-expansive participants and transgender men, respectively: total score $(\alpha=0.71$ and 0.74$)$, DFS $(\alpha=0.81$ and 0.82$)$, AI
( $\alpha=0.78$ and 0.77$)$, and FI ( $\alpha=0.83$ and 0.83 ); for transgender women, internal consistency for the MDDI total and subscale scores ranged from questionable to acceptable: total score $(\alpha=0.67)$, DFS $(\alpha=0.65)$, AI $(\alpha=0.74)$, and FI $(\alpha=0.79)$.

\section{Data analysis}

Results are presented in terms of percentiles, mean (standard deviation), median (interquartile range [IQR]), and percentage. The assumption of normality was not fulfilled among continuous variables (Shapiro-Wilk: $p<.001$ ), so the Kruskal-Wallis test was used to assess gender group differences (gender-expansive people, transgender men, transgender women) and post hoc Dunn's tests were used to examine pairwise differences [28]. The chi-square test was used to compare proportions of participants in each group that had an MDDI total score reflecting clinical significance (based on a cutoff score of $\geq 39$ [23]).

The $R$ statistical environment (RStudio, version 3.6.2; $\mathrm{R}$ Development Core Team, 2019) was used to conduct analyses. The psyche package [29] was used to conduct bivariate analyses. For participants with missing data but greater than $50 \%$ of responses to items, missing values were minimal (gender-expansive people: $0.08 \%$; transgender men: $0.07 \%$; transgender women: $0.02 \%)$. The MissMech package's nonparametric test of homoscedasticity was used to assess the mechanism of missing data [30]. All missing data were consistent with missing completely at random $(p>.05)$; the mice package was used to perform data imputation with chained equations multivariate imputation [31]. A two-tailed threshold of $p<.05$ was used for evaluating significance of the pairwise comparisons.

\section{Results}

Table 2 presents participant sociodemographics. Across all three groups, participants were predominantly White and college-educated with mean BMIs ranging from 28.0 to $29.7 \mathrm{~kg} / \mathrm{m}^{2}$. The mean age for gender-expansive participants and transgender men was similar (30.0 and 30.9 years, respectively), whereas transgender women had a higher mean age of 41.2 years. Norms for the MDDI across the three groups are presented in Table 3.

Significant differences in the MDDI total score and the DFS and FI subscales were observed across gender minority groups $(p<0.001)$; no differences were observed for the AI subscale (Table 4). Specifically, transgender men scored significantly higher than gender-expansive participants and transgender women on the DFS subscale. Gender-expansive participants scored higher on the DFS subscale than transgender women. On the FI subscale, transgender women scored lower than transgender men and gender-expansive participants; however, 
Table 2 Sociodemographic characteristics of gender-expansive people, transgender men, and transgender women from The PRIDE Study

\begin{tabular}{llll}
\hline & Gender-Expansive People & Transgender Men & Transgender Women \\
\hline $\mathrm{N}$ & 1023 & 326 & 177 \\
Sociodemographic characteristics & Mean \pm SD / \% & Mean \pm SD / \% & Mean \pm SD / \% \\
Age, years & $30.0 \pm 9.9$ & $30.9 \pm 9.8$ & $41.2 \pm 15.0$ \\
Race & & & $89.5 \%$ \\
$\quad$ White & $80.7 \%$ & $88.9 \%$ & $0.6 \%$ \\
$\quad$ Asian/Pacific Islander & $2.9 \%$ & $0.3 \%$ & $0.0 \%$ \\
$\quad$ Black/African American & $1.1 \%$ & $2.9 \%$ & $0.0 \%$ \\
$\quad$ Native American & $0.3 \%$ & $0.3 \%$ & $3.5 \%$ \\
$\quad$ Two or more races & $2.7 \%$ & $0.8 \%$ & $6.4 \%$ \\
$\quad$ Another Race & $12.3 \%$ & $6.7 \%$ & $3.9 \%$ \\
Ethnicity: Hispanic/Latino/a & $5.4 \%$ & $3.9 \%$ & $90.6 \%$ \\
Born in the US & $87.6 \%$ & $89.2 \%$ & \\
Educational attainment & & & $56.8 \%$ \\
$\quad$ College degree or higher & $58.1 \%$ & $28.8 \pm 7.4$ & $28.0 \pm 6.4$ \\
Body mass index (BMI), $\mathrm{kg} / \mathrm{m}^{2}$ & $29.7 \pm 8.5$ & & \\
\hline
\end{tabular}

no significant differences were observed between transgender men and gender-expansive participants. Finally, transgender men scored significantly higher on the MDDI total score than gender-expansive participants and transgender women, and gender-expansive participants scored higher than transgender women.

Overall, $6.5 \%$ of gender-expansive participants, $14.7 \%$ of transgender men, and $2.8 \%$ of transgender women had a total score in the clinically significant range $(\geq 39)$, with these proportions reflecting significant group differences $\left(\chi^{2}(2)=30.42, p<.001\right)$. Specifically, pairwise chisquare analyses indicated that a higher proportion of transgender men were in the clinical range than transgender women $\left(\chi^{2}(1)=15.99, p<.001\right.$, OR $=5.92[95 \%$ $\mathrm{CI}=2.31,19.44])$. In addition, a higher proportion of transgender men, when compared with genderexpansive participants, were in the clinical range $\left(\chi^{2}\right.$ $(1)=20.81, p<.001, \mathrm{OR}=2.50 \quad[95 \% \mathrm{CI}=1.65,3.78])$. There was no significant difference between transgender women and gender-expansive participants in terms of clinically significant score ranges $\left(\chi^{2}(1)=2.94, p=.086\right.$, $\mathrm{OR}=0.42[95 \% \mathrm{CI}=0.13,1.06])$.

\section{Discussion}

In this study, we present, for the first time, norms of the MDDI in gender minorities from the United States. Consistent with our expectations, we found that transgender men reported the highest average MDDI total score, followed by gender-expansive people, then transgender women. The gender minority group differences in MDDI total score were driven largely by the DFS subscale and, to a lesser extent, the FI subscale. Notably, there were no significant differences in the AI subscale among the three groups. This study contributes to the scant literature on MD among gender minorities, providing norms for the MDDI and informing a broader understanding of the nature and degree of MD symptoms in gender minority populations in the United States.

\section{Transgender men}

Transgender men reported the highest average MDDI total score and DFS and FI subscale scores. Additionally, transgender men were more likely to score above the clinical cutoff compared to transgender women and gender-expansive participants. These findings are in line with an Italian study by Amodeo and colleagues [11] that found that transgender men reported higher muscularity concerns than non-binary individuals, transgender women, and cisgender women. Transgender men were also found to report an equally high drive for muscularity compared to cisgender men, consistent with gender norms and body image ideals among men, including transgender men, that are commonly focused on muscularity [32]. Motivated in part by a desire to affirm one's identity, transgender men may engage in traditionally masculine muscle-enhancing behaviors such as bodybuilding and fitness [33]. These activities may also involve specific efforts to modify one's body shape to be more consistent with gender-specific body ideals, such that transgender men may work towards building a more muscular chest and reducing 'feminine' fat, especially around the hips [33]. Thus, relative to transgender women and gender-expansive people, the greater severity of MD symptoms among transgender men may be 
Table 3 Distribution of means, standard deviations, medians, interquartile ranges, and percentile ranks for the Muscle Dysmorphic Disorder Inventory (MDDI) among gender-expansive people, transgender men, and transgender women from The PRIDE Study

\begin{tabular}{|c|c|c|c|c|c|c|c|c|c|c|c|c|}
\hline & \multicolumn{4}{|c|}{ Gender-Expansive People $(N=1023)$} & \multicolumn{4}{|c|}{ Transgender Men $(N=326)$} & \multicolumn{4}{|c|}{ Transgender Women $(N=177)$} \\
\hline & $\begin{array}{l}\text { MDDI } \\
\text { DFS }\end{array}$ & $\begin{array}{l}\text { MDDI } \\
\text { Al }\end{array}$ & $\begin{array}{l}\text { MDDI } \\
\mathrm{FI}\end{array}$ & $\begin{array}{l}\text { MDDI } \\
\text { Total }\end{array}$ & $\begin{array}{l}\text { MDDI } \\
\text { DFS }\end{array}$ & $\begin{array}{l}\text { MDDI } \\
\text { Al }\end{array}$ & $\begin{array}{l}\text { MDDI } \\
\mathrm{FI}\end{array}$ & $\begin{array}{l}\text { MDDI } \\
\text { Total }\end{array}$ & $\begin{array}{l}\text { MDDI } \\
\text { DFS }\end{array}$ & $\begin{array}{l}\text { MDDI } \\
\text { Al }\end{array}$ & $\begin{array}{l}\text { MDDI } \\
\mathrm{FI}\end{array}$ & $\begin{array}{l}\text { MDDI } \\
\text { Total }\end{array}$ \\
\hline$M(S D)$ & $8.3(3.9)$ & $12.8(4.0)$ & $6.1(3.1)$ & $27.2(6.7)$ & $10.7(4.5)$ & $13.2(4.0)$ & $6.6(3.3)$ & $30.5(7.5)$ & $6.1(2.2)$ & $13.0(4.0)$ & $5.5(2.6)$ & $24.6(5.7)$ \\
\hline Range & $5-24$ & $4-20$ & $4-20$ & $13-56$ & $5-25$ & $4-20$ & $4-20$ & $13-57$ & $5-22$ & $4-20$ & $4-16$ & $14-43$ \\
\hline \multicolumn{13}{|c|}{ Percentile rank } \\
\hline 5 & 5.0 & 6.0 & 4.0 & 17.0 & 5.0 & 6.0 & 4.0 & 20.0 & 5.0 & 6.0 & 4.0 & 16.0 \\
\hline 10 & 5.0 & 7.0 & 4.0 & 19.0 & 5.0 & 7.5 & 4.0 & 22.0 & 5.0 & 7.0 & 4.0 & 18.0 \\
\hline 15 & 5.0 & 8.0 & 4.0 & 20.0 & 6.0 & 9.0 & 4.0 & 23.0 & 5.0 & 9.0 & 4.0 & 19.0 \\
\hline 20 & 5.0 & 9.0 & 4.0 & 21.0 & 7.0 & 10.0 & 4.0 & 25.0 & 5.0 & 10.0 & 4.0 & 20.0 \\
\hline 25 & 5.0 & 10.0 & 4.0 & 22.0 & 7.0 & 10.3 & 4.0 & 25.0 & 5.0 & 10.0 & 4.0 & 21.0 \\
\hline 30 & 5.0 & 11.0 & 4.0 & 23.0 & 8.0 & 11.0 & 4.0 & 26.0 & 5.0 & 11.0 & 4.0 & 21.0 \\
\hline 35 & 6.0 & 11.0 & 4.0 & 24.0 & 8.0 & 12.0 & 4.0 & 27.0 & 5.0 & 11.0 & 4.0 & 22.0 \\
\hline 40 & 6.0 & 12.0 & 4.0 & 25.0 & 9.0 & 12.0 & 4.0 & 28.0 & 5.0 & 11.4 & 4.0 & 22.4 \\
\hline 45 & 7.0 & 12.0 & 4.0 & 26.0 & 9.0 & 13.0 & 4.3 & 29.0 & 5.0 & 12.0 & 4.0 & 23.0 \\
\hline 50 & 7.0 & 13.0 & 4.0 & 27.0 & 10.0 & 13.0 & 5.0 & 30.0 & 5.0 & 13.0 & 4.0 & 24.0 \\
\hline 55 & 8.0 & 14.0 & 5.0 & 27.0 & 11.0 & 14.0 & 6.0 & 31.0 & 5.0 & 13.0 & 4.0 & 25.0 \\
\hline 60 & 8.0 & 14.0 & 5.0 & 28.0 & 12.0 & 14.0 & 6.0 & 31.0 & 5.0 & 14.0 & 4.0 & 25.6 \\
\hline 65 & 9.0 & 15.0 & 6.0 & 29.0 & 12.0 & 15.0 & 7.0 & 33.0 & 6.0 & 15.0 & 5.0 & 26.0 \\
\hline 70 & 9.0 & 15.0 & 7.0 & 30.0 & 12.0 & 16.0 & 8.0 & 33.5 & 6.0 & 15.2 & 5.0 & 27.0 \\
\hline 75 & 10.0 & 16.0 & 8.0 & 31.0 & 13.0 & 17.0 & 8.0 & 35.0 & 7.0 & 16.0 & 6.0 & 28.0 \\
\hline 80 & 11.0 & 17.0 & 8.0 & 32.0 & 14.0 & 17.0 & 9.0 & 36.0 & 7.0 & 17.0 & 6.8 & 29.0 \\
\hline 85 & 12.0 & 17.0 & 9.0 & 34.0 & 15.0 & 18.0 & 10.0 & 38.0 & 8.0 & 18.0 & 8.0 & 31.0 \\
\hline 90 & 14.0 & 18.0 & 11.0 & 37.0 & 17.5 & 18.0 & 11.0 & 41.0 & 8.0 & 18.4 & 9.4 & 31.4 \\
\hline 95 & 16.0 & 19.0 & 13.0 & 39.0 & 20.0 & 19 & 13.0 & 44.8 & 9.0 & 19.0 & 12.0 & 34.2 \\
\hline 99 & 21.0 & 20.0 & 16.0 & 45.0 & 23.8 & 20.0 & 17.8 & 48.8 & 15.2 & 20.0 & 16.0 & 40.2 \\
\hline
\end{tabular}

MDDI Muscle Dysmorphic Disorder Inventory, MDDI DFS MDDI Drive for Size subscale, MDDI AI MDDI Appearance Intolerance subscale, MDDI FI MDDI Functional Impairment subscale, $M$ Mean, $S D$ standard deviation

uniquely intertwined with the affirmation of the male identity and pressures to conform to masculine norms $[34,35]$. Whereas traditional eating disorder symptoms oriented toward thinness and weight loss, along with the concomitant loss of menses and feminine body shape, are purportedly intertwined with the construction of a female identity [34], a hegemonic masculine identity is often conflated with the presence of muscularity [36]. As such, and in keeping with our findings, the affirmation of a male identity among transgender men may pose

Table 4 Comparisons of Muscle Dysmorphic Disorder Inventory (MDDI) Total and subscale scores among gender-expansive people, transgender men, and transgender women in The PRIDE Study

\begin{tabular}{|c|c|c|c|c|c|c|}
\hline & \multicolumn{3}{|l|}{ Groups } & \multicolumn{2}{|c|}{ Kruskal-Wallis Test } & \multirow[t]{3}{*}{ post hoc Dunn's test } \\
\hline & Gender Expansive People (a) & Transgender Men (b) & Transgender Women (c) & $\overline{x^{2}}$ & $p$ & \\
\hline & Mdn (IQR) & Mdn (IQR) & Mdn (IQR) & & & \\
\hline MDDI DFS & $7(19)$ & $10(20)$ & $5(17)$ & 197.15 & $<.001$ & $b>a>c$ \\
\hline MDDI Al & $13(16)$ & $13(16)$ & $13(16)$ & 2.05 & .358 & - \\
\hline MDDI FI & $4(16)$ & $5(16)$ & $4(12)$ & 16.48 & $<.001$ & $b, a>c$ \\
\hline MDDI Total & $27(43)$ & $30(44)$ & $24(29)$ & 87.50 & $<.001$ & $b>a>c$ \\
\hline
\end{tabular}

MDDI Muscle Dysmorphic Disorder Inventory, MDDI DFS Drive for Size subscale, MDDI AI Appearance Intolerance subscale, MDDI FI Functional Impairment subscale 
unique vulnerabilities with regard to muscularityoriented psychopathology.

\section{Transgender women}

We found that transgender women had the lowest average MDDI total score and DFS subscale score, which aligns with prior research [11]. This finding also accords with prior studies showing lower MD symptoms, particularly DFS, among cisgender women [11, 23]. Transgender women may experience lower muscularity concerns due to feminine body norms placing greater emphasis on thinness or on muscle tone versus bulk [12, 37]. As the MDDI was initially developed in men and the construct of MD more generally emphasizes muscle size versus definition or leanness, the items may have differential relevance for women. This may also explain the lower internal consistency of the MDDI total scale and the DFS subscale among transgender women relative to transgender men and gender-expansive people, respectively. Future work will need to be conducted to better understand potentially unique considerations regarding the nature and assessment of MD symptoms among transgender women. For instance, a future study could concurrently examine both drive for thinness and drive for muscularity among transgender women and transgender men to elucidate how the constructs may differentially relate to mental health concerns across these groups.

\section{Gender-expansive people}

Overall, gender-expansive people reported average scores on the MDDI that were intermediate to those of transgender men and transgender women. We found a greater range and variability in MDDI scores in this group relative to transgender men and women, which is consistent with prior findings in this population, thus indicating heterogeneity in body image concerns, including in regards to overall body shape and specific body parts such as chest size and genitals [38]. Some gender-expansive people also expressed a drive to achieve an "androgynous or fluid" body ideal, or a balance of masculine and feminine traits [38]. This is consistent with the current finding that scores on the AI subscale did not significantly differ across the three gender minority groups. Moreover, gender-expansive individuals may be disproportionately exposed to certain social stressors, interpersonal violence, and psychological comorbidities (such as self-harm behaviors), relative to cisgender and binary transgender men and women, which in turn may elevate their risk for body image concerns [13]. As such, research on body image, disturbance to body image, and related risk and protective factors are warranted in this population.

\section{Comparisons to prior studies}

Compared to the initial MDDI validation study in a selected sample of presumably cisgender men weightlifters [16], our community sample of TGE people reported qualitatively higher MDDI total scores (24.6-30.5 vs 18.8 ) and AI subscale scores (12.8-13.2 vs 6.1), but similar DFS (6.1-10.7 vs. 7.5) and FI (5.5-6.6 vs. 6.4) subscale scores. A prior German study of presumably cisgender populations recruited through fitness and bodybuilding groups found that $25 \%$ of men and $16 \%$ of women scored above the same MDDI clinical cutoff used in this study [23], which are qualitatively higher than the clinical cutoffs we found among a community sample of TGE populations. However, these comparisons with presumably cisgender populations should be interpreted with caution given that people participating in fitness, bodybuilding, or weightlifting may be at greater risk for MD symptoms than the general population.

\section{Gender minority stress framework}

While gender minority populations may differentially experience MD symptoms, such as those oriented toward a DFS, we found no significant differences in AI. One explanation for this may be the shared experience of discrimination, stigma, and prejudice that TGE individuals encounter secondary to society's intolerance of their gender identity, termed the gender minority stress framework $[9,39]$. Specifically, gender minority individuals may face psychological distress due to societal identity invalidation, decreased social support, and increased discrimination [40-42]. Many TGE individuals experience mistreatment and violence, as evidenced by results from the 2015 U.S. Transgender Survey, in which $46 \%$ of the participants reported verbal harassment and $9 \%$ reported being physically attacked due to their gender identity in the prior year [43]. Experiences of gender dysphoria may also play a role in AI, particularly with regard to discomfort with one's body, as reflected in the MDDI item "I hate my body." Of note, some studies have shown a decrease in body image disturbance and eating disorder symptoms among individuals who receive gender-affirming health care [44, 45]; thus, examining the effect of gender-affirming health care on MD symptoms is an important area of future research.

\section{Limitations}

Certain limitations of the current study should be noted. First, access to or engagement in gender-affirming health care was not known in the current sample. Second, participants in this study were predominantly White and highly educated; thus, results may not be generalizable to all gender minority people in the United States, particularly those from racial or ethnic minority backgrounds. Third, this study was cross-sectional and did 
not assess the onset or duration of MD symptoms. Future prospective, longitudinal studies are therefore recommended. Fourth, the current study only administered the MDDI, which is one of several MD symptom measures (e.g., the Muscle Dysmorphia Inventory [14], Muscle Appearance Satisfaction Scale [15]). Thus, we were not able to evaluate whether the MDDI is the best measure for assessing MD symptoms in gender minority populations. Fifth, we did not conduct a psychometric validation as part of the current study, and future research is needed to psychometrically evaluate the MDDI in gender minority groups. Sixth, although we used a previously published cutoff for clinical significance [23], this cutoff was not developed specifically in gender minority populations. Nonetheless, strengths of the study include a relatively large and unique sample of populations that have been understudied, particularly within the MD literature.

\section{Conclusions}

We report, for the first time, MDDI norms among gender-expansive people, transgender men, and transgender women. Given the increasingly recognized health disparities that affect gender minority individuals, establishing normative data on MD symptoms will facilitate the ability for clinicians and researchers to interpret MDDI scores in these understudied populations. Future research will be needed to examine the MDDI in gender minorities clinically diagnosed with MD or MD measures tailored to specific gender minority populations. Nationally representative, population-based research with the MDDI is needed to better approximate the prevalence of MD and factors associated with MD in diverse communities. Future research could examine if TGE individuals differ on overall body image/size dissatisfaction versus body dysmorphic symptoms (e.g., genital size dissatisfaction) and potential cascading effects of these differences. Furthermore, the important intersections of sociodemographic factors - such as race, ethnicity, age, and socioeconomic status - as well as gender minority stressors on the nature and degree of $\mathrm{MD}$ symptoms and mental health outcomes among gender minority populations warrants additional study.

\section{Abbreviations \\ Al: Appearance Intolerance subscale; BMI: Body mass index; DFS: Drive for Size subscale; DSM-5: Fifth Edition of the Diagnostic and Statistical Manual of Mental Disorders; Fl: Functional Impairment subscale; M: Mean; MD: Muscle Dysmorphia; MDDI: Muscle Dysmorphic Disorder Inventory; PRIDE Study: Population Research in Identities and Disparities for Equality (PRIDE) Study; SD: Standard deviation; TGE: Transgender and gender-expansive}

\section{Acknowledgments}

We thank Samuel E. Benabou and Kelsey Wu for editorial assistance. The PRIDE Study is a community-engaged research project that serves and is made possible by LGBTQ+ community involvement at multiple points in the research process, including the dissemination of findings. We acknowledge the courage and dedication of The PRIDE Study participants for sharing their stories, the careful attention of the PRIDEnet Participant Advisory Committee (PAC) members for reviewing and improving every study application, and the enthusiastic engagement of the PRIDEnet Ambassadors and Community Partners for bringing thoughtful perspectives as well as promoting enrollment and disseminating findings. For more information, please visit https:// pridestudy.org/pridenet.

\section{Authors' contributions}

Jason M. Nagata: Conceptualization, Formal analysis, Methodology, Writing original draft, Writing - review \& editing. Emilio J. Compte: Formal analysis, Methodology, Writing - original draft, Writing - review \& editing. F. Hunter McGuire: Writing - original draft, Writing - review \& editing. Jason M. Lavender: Conceptualization, Writing - review \& editing. Tiffany A. Brown: Conceptualization, Writing - review \& editing. Stuart B. Murray:

Conceptualization, Writing - review \& editing. Annesa Flentje:

Conceptualization, Methodology, Writing - review \& editing. Matthew R. Capriotti: Conceptualization, Methodology, Writing - review \& editing. Micah E. Lubensky: Conceptualization, Methodology, Writing - review \& editing. Juno Obedin-Maliver: Conceptualization, Methodology, Writing - review \& editing. Mitchell R. Lunn: Conceptualization, Methodology, Writing - review \& editing. The author(s) read and approved the final manuscript.

\section{Funding}

JMN is supported by the American Heart Association Career Development Award (CDA34760281) and was a participant in the Pediatric Scientist Development Program that was supported by the American Academy of Pediatrics and American Pediatric Society. SBM was supported by the National Institutes of Health (K23 MH115184). AF was supported by the National Institute on Drug Abuse (K23DA039800). MC was supported by a Clinical Research Training Fellowship from the American Academy of Neurology and the Tourette Association of America. Research reported in this article was partially funded through a Patient-Centered Outcomes Research Institute (www.pcori.org; PPRN-1501-26848) to MRL. JOM was partially supported by K12DK111028 from the National Institute of Diabetes, Digestive, and Kidney Disorders. The statements in this article are solely the responsibility of the authors and do not necessarily represent the views of PatientCentered Outcomes Research Institute, its Board of Governors or Methodology Committee, the Uniformed Services University, the Department of Defense, or the National Institutes of Health.

\section{Availability of data and materials}

Data from The PRIDE Study may be accessed through an Ancillary Study application (details at pridestudy.org/collaborate).

\section{Declarations}

\section{Ethics approval and consent to participate}

The University of California, San Francisco and Stanford University Institutional Review Boards approved this study (\#16-21213). Written informed consent was obtained from all participants.

\section{Consent for publication}

Not applicable.

\section{Competing interests}

Dr. Juno Obedin-Maliver has consulted for Sage Therapeutics (5/2017) in a one-day advisory board, Ibis Reproductive Health (a non-for-profit research group; 3/2017-5/2018, 2020-present), Folx, Inc. (2020 - present), and Hims Inc. (2019 - present). Dr. Lunn has consulted for Hims Inc. (2019 - present) and Folx, Inc. (2020). None of these roles present a conflict of interest with this work as described here. The other authors have no conflicts of interest to report.

\section{Author details}

${ }^{1}$ Department of Pediatrics, University of California, 550 16th Street, 4th Floor, Box 0110, San Francisco, CA 94158, USA. Eating Behavior Research Center, School of Psychology, Universidad Adolfo Ibáñez, Santiago, Chile. ${ }^{3}$ Research Department, Comenzar de Nuevo Treatment Center, Monterrey, Mexico. ${ }^{4}$ The Brown School, Washington University in St. Louis, St. Louis, MO, USA. ${ }^{5}$ Military Cardiovascular Outcomes Research Program (MiCOR), Department 
of Medicine, Uniformed Services University of the Health Sciences, Bethesda, MD, USA. ${ }^{6}$ The Metis Foundation, San Antonio, TX, USA. ${ }^{7}$ Department of Psychiatry, University of California, San Diego, CA, USA. ${ }^{8}$ San Diego State University Research Foundation, San Diego, CA, USA. ${ }^{9}$ Department of Psychiatry and Behavioral Sciences, University of Southern California, Los Angeles, CA, USA. ${ }^{10}$ Department of Community Health Systems, University of California, San Francisco, San Francisco, CA, USA. ${ }^{11}$ Alliance Health Project, Department of Psychiatry and Behavioral Sciences, University of California, San Francisco, San Francisco, CA, USA. ${ }^{12}$ The PRIDE Study/PRIDEnet, Stanford University School of Medicine, Stanford, CA, USA. ${ }^{13}$ Department of Psychology, San José State University, San Jose, CA, USA. ${ }^{14}$ Department of Obstetrics and Gynecology, Stanford University School of Medicine, Stanford, CA, USA. ${ }^{15}$ Department of Epidemiology and Population Health, Stanford University School of Medicine, Stanford, CA, USA. ${ }^{16}$ Division of Nephrology, Department of Medicine, Stanford University School of Medicine, Stanford, CA, USA.

Received: 12 April 2021 Accepted: 2 July 2021

Published online: 14 July 2021

\section{References}

1. American Psychiatric Association. Diagnostic and Statistical Manual of Mental Disorders Fifth Edition. Arlington: American Psychiatric Association: 2013.

2. Pope CG, Pope HG, Menard W, Fay C, Olivardia R, Phillips KA. Clinical features of muscle dysmorphia among males with body dysmorphic disorder. Body Image. 2005;2(4):395-400. https://doi.org/10.1016/j.bodyim.2 005.09.001.

3. Badenes-Ribera L, Rubio-Aparicio M, Sánchez-Meca J, Fabris MA, Longobardi C. The association between muscle dysmorphia and eating disorder symptomatology: A systematic review and meta-analysis. J. Behav. Addict. Akademiai Kiado Rt:; 2019. p. 351-71.

4. Cafri G, Olivardia R, Thompson JK. Symptom characteristics and psychiatric comorbidity among males with muscle dysmorphia. Compr Psychiatry. 2008:49(4):374-9. https://doi.org/10.1016/j.comppsych.2008.01.003.

5. Mitchell L, Murray SB, Cobley S, Hackett D, Gifford J, Capling L, et al. Muscle dysmorphia symptomatology and associated psychological features in bodybuilders and non-bodybuilder resistance trainers: a systematic review and meta-analysis. Sport Med. 2017:47(2):233-59. https://doi.org/10.1007/s4 0279-016-0564-3.

6. Santos Filho CA dos, Tirico PP, Stefano SC, Touyz SW, Claudino AM. Systematic review of the diagnostic category muscle dysmorphia. Aust N Z J Psychiatry. 2016;50(4):322-33. https://doi.org/10.1177/0004867415614106.

7. Sandgren SS, Lavallee D. Muscle dysmorphia research neglects DSM-5 diagnostic criteria. J Loss Trauma Routledge. 2018;23(3):211-43. https://doi. org/10.1080/15325024.2018.1428484.

8. White Hughto JM, Reisner SL, Pachankis JE. Transgender stigma and health: A critical review of stigma determinants, mechanisms, and interventions. Soc Sci Med. 2015;147:222-31. https://doi.org/10.1016/j.socscimed.2015.11. 010. Epub 2015 Nov 11.

9. Hendricks ML, Testa RJ. A conceptual framework for clinical work with transgender and gender nonconforming clients: an adaptation of the minority stress model. Prof Psychol Res Pract. 2012;43(5):460-7. https://doi. org/10.1037/a0029597.

10. Testa RJ, Habarth J, Peta J, Balsam K, Bockting W. Development of the Gender Minority Stress and Resilience Measure. Psychol Sex Orientat Gend Divers. 2015;2:65-77.

11. Amodeo AL, Esposito C, Antuoni S, Saracco G, Bacchini D. Muscle dysmorphia: what about transgender people? Cult Heal Sex. 2020. https:// doi.org/10.1080/13691058.2020.1814968.

12. Witcomb GL, Bouman WP, Brewin N, Richards C, Fernandez-Aranda F, Arcelus $J$. Body image dissatisfaction and eating-related psychopathology in trans individuals: a matched control study. Eur Eat Disord Rev J Eat Disord Assoc. 2015;23(4):287-93. https://doi.org/10.1002/erv.2362.

13. Lefevor GT, Boyd-Rogers CC, Sprague BM, Janis RA. Health disparities between genderqueer, transgender, and cisgender individuals: An extension of minority stress theory. J Couns Psychol. 2019;66:385-95.

14. Rhea DJ, Lantz CD, Cornelius AE. Development of the Muscle Dysmorphia Inventory (MDI). J Sports Med Phys Fitness. 2004:44:428-35 Available from: https://europepmc.org/article/med/15758857, [cited 2021 May 21].
15. Mayville SB, Williamson DA, White MA, Netemeyer RG, Drab DL. Development of the muscle appearance satisfaction scale: A self-report measure for the assessment of muscle dysmorphia symptoms. Assessment. 2002:9:351-60. https://doi.org/10.1177/1073191102238156.

16. Hildebrandt T, Langenbucher J, Schlundt DG. Muscularity concerns among men: development of attitudinal and perceptual measures. Body Image. 2004;1 (2):169-81. https://doi.org/10.1016/j.bodyim.2004.01.001.

17. Compte E, Nagata JM, Sepúlveda AR, Rivas A, Sbdar LS, Menga S, et al. Assessment and validation of a Spanish version of the muscle dysmorphia disorder inventory in Argentinian men who exercise: Inventario de Dismorfia muscular. Body Image. 2019;31:24-34. https:/doi.org/10.1016/.bodyim.2019.08.002.

18. Galiana-Llinares L, Badenes-Rivera L, Fuentes-Durán MC. Validación de la versión española del Inventario del Trastorno por Dismorfia Muscular en una muestra de estudiantes universarios. In: Scinfoper, editor. editor. Atención a las necesidades comunitarias para la salud; 2017. p. 41-5.

19. Gomes VMGM, Compte EJ, Almeida M, Campos PF, Queiroz ACC, Pereira LF, et al. Psychometric Properties of the Muscle Dysmorphic Disorder Inventory Among Physically Active Brazilian College Men. Psychol Men Masculinity. 2020;21(4):622-31. https://doi.org/10.1037/men0000307.

20. Sandgren SS, Giske R, Shalfawi SAl. Muscle dysmorphia in norwegian gymgoing men: an initial investigation. Kinesiology. 2019;51(1):12-21. https://doi. org/10.26582/k.51.1.3.

21. Santarnecchi E, Dèttore D. Muscle dysmorphia in different degrees of bodybuilding activities: validation of the Italian version of muscle dysmorphia disorder inventory and bodybuilder image grid. Body Image. 2012;9(3):396-403. https://doi.org/10.1016/j.bodyim.2012.03.006.

22. Sepúlveda AR, Rica R, Moreno A, Román FJ, Compte EJ. Asessing the male body image: Spanish validation of two instruments. Psychiatry. 2019;272: $483-90$.

23. Zeeck A, Welter V, Alatas $H$, Hildebrandt $T$, Lahmann C, Hartmann A. Muscle Dysmorphic Disorder Inventory (MDDI): Validation of a German version with a focus on gender. PLoS One. 2018;13(11):e0207535. https://doi.org/10.1371/ journal.pone.0207535.

24. Compte EJ, Cattle CJ, Lavender JM, Murray SB, Brown TA, Capriotti MR, et al. Psychometric evaluation of the Muscle Dysmorphic Disorder Inventory (MDDI) among cisgender gay men and cisgender lesbian women. Body Image. 2021;38:241-50 Available from: https://linkinghub.elsevier.com/ retrieve/pii/S1740144521000619. [cited 2021 May 19].

25. Lunn MR, Capriotti MR, Flentje A, Bibbins-Domingo K, Pletcher MJ, Triano $\mathrm{AJ}$, et al. Using mobile technology to engage sexual and gender minorities in clinical research. Plos One. 2019;14(5):e0216282. https://doi.org/10.1371/ journal.pone.0216282.

26. Lunn MR, Lubensky M, Hunt C, Flentje A, Capriotti MR, Sooksaman C, et al. A digital health research platform for community engagement, recruitment, and retention of sexual and gender minority adults in a national longitudinal cohort study--The PRIDE Study. J Am Med Informatics Assoc JAMIA. 2019;26(8-9):737-48. https://doi.org/10.1093/jamia/ocz082.

27. Strübel J, Petrie TA. Appearance and performance enhancing drug usage and psychological well-being in gay and heterosexual men. Psychol Sex Routledge. 2019;10:132-48.

28. Katz MH. Study design and statistical analysis: a practical guide for clinicians. Study Des. Stat. Anal. A Pract. Guid. Clin. Cambridge University Press; 2006, doi: https://doi.org/10.1017/CBO9780511616761.

29. Revelle W. psych: Procedures for Personality and Psychological Research. Evanston: Northwestern University; 2018. https://CRAN.R-project.org/package=psych.

30. Jamshidian $\mathrm{M}$, Jalal $\mathrm{S}$, Jansen C. Missmech: an R package for testing homoscedasticity, multivariate normality, and missing completely at random (MCAR). J Stat Softw. 2014;56:1-31.

31. van Buuren $\mathrm{S}$, Groothuis-Oudshoorn K. mice: Multivariate imputation by chained equations in R. J Stat Softw. 2011:45:1-67.

32. Nagata JM, Domingue BW, Darmstadt GL, Weber AM, Meausoone V, Cislaghi B, et al. Gender norms and weight control behaviors in U.S. adolescents: a prospective cohort study (1994-2002). J Adolesc Heal Off Publ Soc Adolesc Med. 2020;66(1):S34-41. https://doi.org/10.1016/j.jadohea Ith.2019.08.020.

33. Farber R. Transing' fitness and remapping transgender male masculinity in online message boards. J Gend Stud. 2017;26:254-68. https://doi.org/10.1 080/09589236.2016.1250618

34. Murray SB. Gender identity and eating disorders: the need to delineate novel pathways for eating disorder symptomatology. J Adolesc Health. 2017;60(1):1-2. https://doi.org/10.1016/j.jadohealth.2016.10.004. 
35. Vegter V. Conceptualizing Masculinity in Female-to-Male Trans-Identified Individuals: A Qualitative Inquiry Conceptualisation de la masculinité chez les personnes à transidentité femme-homme : une enquête qualitative. Can J Couns Psychother. 2013;47(1). https://cjc-rcc.ucalgary.ca/article/view/60923.

36. Murray SB, Touyz SW. Masculinity, femininity and male body image: a recipe for future research. Int J Mens Health. 2012;11(3):227-39. https://doi.org/1 0.3149/jmh.1103.227.

37. Rodgers RF, Franko DL, Lovering ME, et al. Development and Validation of the Female Muscularity Scale. Sex Roles. 2018;78(2):18-26. https://doi.org/1 0.1007/s11199-017-0775-6.

38. Galupo MP, Pulice-Farrow L, Pehl E. "There Is Nothing to Do About It": Nonbinary Individuals' Experience of Gender Dysphoria. Transgender Heal. 2021;6(2):101-110. http://doi.org/10.1089/trgh.2020.0041.

39. Tan KKH, Treharne GJ, Ellis SJ, Schmidt JM, Veale F. Gender minority stress : a critical review. J Homosex. 2020;67:1471-89. https://doi.org/10.1080/0091 8369.2019.1591789.

40. Scandurra C, Mezza F, Maldonato NM, Bottone M, Bochicchio V, Valerio P, et al. Health of non-binary and genderqueer people: A systematic review. Front Psychol. 2019;10:1453.

41. Aparicio-García ME, Díaz-Ramiro EM, Rubio-Valdehita S, López-Núñez Ml, García-Nieto I. Health and well-being of cisgender, transgender and nonbinary young people. Int J Environ Res Public Health. 2018;15(10):2133. https://doi.org/10.3390/ijerph15102133.

42. Johnson KC, Leblanc AJ, Deardorff J, Bockting WO, Johnson KC, Leblanc AJ, et al. Invalidation experiences among non-binary adolescents invalidation experiences among non-binary adolescents. J Sex Res. 2020;57:222-33. https://doi.org/10.1080/00224499.2019.1608422.

43. James SE, Herman JL, Rankin S, Keisling M, Mottet L, Anafi M. The Report of the 2015 U.S. Transgender Survey. 2016. Available from: https:/transequality. org/sites/default/files/docs/usts/USTS-Full-Report-Dec17.pdf

44. Jones BA, Haycraft E, Bouman WP, Brewin N, Claes L, Arcelus J. Risk Factors for Eating Disorder Psychopathology within the Treatment Seeking Transgender Population: The Role of Cross-Sex Hormone Treatment. Eur Eat Disord Rev. 2018;26:120-8 Available from: http://www.ncbi.nlm.nih.gov/ pubmed/29318711. [cited 2020 mar 16].

45. Testa RJ, Rider GN, Haug NA, Balsam KF. Gender confirming medical interventions and eating disorder symptoms among transgender individuals. Heal Psychol Off J Div Heal Psychol Am Psychol Assoc. 2017;36: 927-36

\section{Publisher's Note}

Springer Nature remains neutral with regard to jurisdictional claims in published maps and institutional affiliations.

Ready to submit your research? Choose BMC and benefit from:

- fast, convenient online submission

- thorough peer review by experienced researchers in your field

- rapid publication on acceptance

- support for research data, including large and complex data types

- gold Open Access which fosters wider collaboration and increased citations

- maximum visibility for your research: over $100 \mathrm{M}$ website views per year

At $\mathrm{BMC}$, research is always in progress.

Learn more biomedcentral.com/submissions 